\title{
Long-term follow-up evaluation of magnetic resonance imaging in the prognosis of permanent GH deficiency
}

\author{
Mauro Bozzola, Fabio Mengarda ${ }^{1}$, Paola Sartirana, Luciano Tatò ${ }^{1}$ and J L Chaussain ${ }^{2}$ \\ Dipartimento di Scienze Pediatriche, Università di Pavia, IRCCS Policlinico San Matteo, Piazzale C Golgi 2, 27100 Pavia, Italy, \\ ${ }^{1}$ Dipartimento di Pediatria, Università di Verona, Ospedale Borgoroma, 37100 Verona, Italy and ${ }^{2}$ Hôpital Saint Vincent-de-Paul, \\ 81 avenue Denfert-Rochereau, Paris, France \\ (Correspondence should be addressed to M Bozzola; Email: m.bozzola@smatteo.pv.it)
}

\begin{abstract}
Objective: In patients with GH deficiency (GHD), magnetic resonance imaging (MRI) has revealed morphological abnormalities such as pituitary hypoplasia, pituitary stalk agenesis (PSA) and ectopia of the posterior pituitary (PPE). The MRI anomalies have been more frequently reported in patients with multiple pituitary hormone deficiency (MPHD) than in subjects with isolated GH deficiency (IGHD). The aim of this work was to define which MRI anatomical abnormalities of the hypothalamo-pituitary area can be considered as a prognostic marker of permanent GHD.

Design: To investigate the relationship between the neuroradiological images and endocrine findings, we clinically re-evaluated 93 out of the 121 GHD patients with IGHD and MPHD previously studied. Results: No additional hormone deficiencies were observed in 55 out of 60 patients initially classified as having IGHD with a normal (15 cases) or reduced (40 cases) pituitary gland size, without other MRI abnormalities. The remaining five children, who had initially shown an apparently IGHD in spite of PSA and PPE, developed a MPHD over time. In 33 MPHD patients with (25 cases) or without (8 cases) MRI abnormalities, the associated hormone deficiencies were confirmed during follow-up.

Conclusions: The IGHD patients showing PSA and PPE inevitably develop additional hormone deficiencies, while IGHD subjects having no MRI abnormalities maintain IGHD. Moreover, the anatomical abnormalities of the hypothalamo-pituitary area can be considered as a prognostic marker of permanent GHD.
\end{abstract}

European Journal of Endocrinology 143 493-496

\section{Introduction}

In the last few years, magnetic resonance imaging (MRI) has dramatically improved the diagnostic accuracy of the evaluation of the hypothalamo-pituitary region. Morphological and structural abnormalities can be observed in patients with growth hormone deficiency (GHD) using the neuroradiological study (1). Typical pathological features revealed by MRI basically include: (i) severe pituitary hypoplasia (i.e. gland height at less than -2 s.D. for age), (ii) pituitary stalk agenesis (PSA), and (iii) lack of the normal T1-weighted posterior pituitary hyperintensity in the posterior part of the sella turcica and presence of a high-intensity signal at the infundibular level (posterior pituitary ectopia, PPE). The MRI anomalies have been more frequently reported in patients with multiple pituitary hormone deficiency (MPHD) than in subjects with isolated GH deficiency (IGHD) (2-7). However, it is unclear whether the pathological features detected by MRI are invariably associated with permanent GHD.
The aim of our study was to define which MRI anatomical abnormalities of the hypothalamo-pituitary area can be considered as a prognostic marker of permanent GHD.

\section{Subjects and methods}

In a previous study (3), MRI was evaluated in 121 patients with IGHD (81 cases) and MPHD (40 cases), aged 0.3-25 years, who were followed at the Paediatric Department of the Policlinico San Matteo, Pavia, Italy and the Hospital Saint Vincent-de-Paul, Paris. In the present study, 93 out of the 121 GHD patients, who were regularly evaluated every 3 or 4 months, were examined 6 years later. Unfortunately, the remaining 28 subjects were missed during follow-up because of practical reasons, e.g. they moved to other towns or discontinued early GH therapy. French and Italian MRI studies were performed with a 1-5-T superconducting system (Magnetom, Siemens, Germany). A conventional 


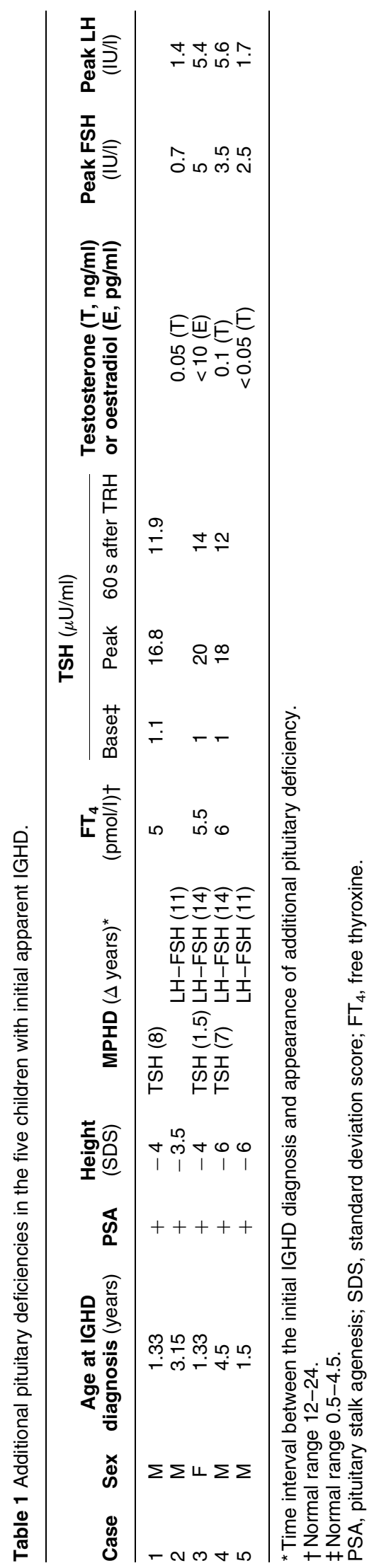

spin-echo pulse sequence was adopted using standard pituitary protocol. Sagittal and coronal T1-weighted images (TR, $400 \mathrm{~ms}$; TE, $15 \mathrm{~ms}$; three excitations) were obtained using $3 \mathrm{~mm}$ thick sections (matrix size, $256 \times 256$ pixels; $20 \mathrm{~cm}$ field of view). The spatial resolution was $2-3 \mathrm{~mm}$. The total acquisition time was $5 \mathrm{~min}$. The pituitary height, which better reflects the gland growth, was determined on the mid-sagittal T1weighted image by measuring the greatest distance between the base and the top of the gland using a cursor on the display (8). Pituitary height measurements were compared with published normal values for age as obtained using the same imaging and laboratory studies (8). A pituitary was considered to be hypoplastic when the gland height was at less than -2 S.D. for age.

Each MR image was evaluated independently by both experienced French and Italian observers who agreed in their assessment of the studied parameters. A board certified neuroradiologist determined the manner in which measurements were made and reviewed the neuroradiological evaluations.

The pituitary-thyroid axis function was investigated by evaluation of serum free thyroxine $\left(\mathrm{T}_{4}\right)$ and thyrotrophin (TSH) values, and by thyrotrophinreleasing hormone (TRH) test $\left(200 \mu \mathrm{g} / \mathrm{m}^{2}\right.$ i.v. $)$ : low free $\mathrm{T}_{4}$ values with a delayed and sustained response of TSH following TRH injection were considered as hypothalamic hypothyroidism. Adrenal function was evaluated by morning $(0800 \mathrm{~h})$ and evening $(2000 \mathrm{~h})$ serum cortisol concentrations. Hypothalamo-pituitary-gonadal axis was investigated by gonadotrophin-releasing hormone (GnRH) test (100 $\mu \mathrm{g} / \mathrm{m}^{2}$ i.v.): absence of physical signs of pubertal development beyond a bone age of 12.5 years in girls and 13.5 years in boys, associated with follicle-stimulating hormone (FSH) and luteinizing hormone (LH) basal values at the dosing limit and no increase after GnRH injection was defined as hypogonadotrophic hypogonadism.

Out of the 93 subjects, 60 cases with IGHD, aged 11.8320 years, showed signs of puberty, while the remaining 33 MPHD patients, aged 11.7-29 years, were developing spontaneous or drug-induced puberty (all except a 8.83-year-old boy having GH and TSH deficiency).

\section{Results}

On the basis of clinical and endocrinological findings, no additional hormone deficiencies were observed in 55 out of 60 patients initially classified as having IGHD, with normal (15 cases) or reduced (40 cases) pituitary gland, without other MRI abnormalities. Interestingly, the remaining five children, who had initially shown an apparently IGHD in spite of PSA and PPE, developed a MPHD with time (Table 1). In particular, one of them developed only TSH deficiency, two cases LH-FSH deficiency and the remaining two patients both TSH and LH-FSH deficiency (Table 1). In 33 MPHD patients with ( 25 cases) or without (8 cases) MRI abnormalities, 
Table 2 MRI findings of the hypothalamo-pituitary region in IGHD and MPHD patients.

\begin{tabular}{|c|c|c|}
\hline & IGHD $(n=60)$ & MPHD $(n=33)$ \\
\hline \multicolumn{3}{|l|}{ Pituitary stalk } \\
\hline Normal & $55(91.7 \%)$ & $8(24.3 \%)$ \\
\hline Abnormal & $5(8.3 \%)$ & 25 (75.7\%) \\
\hline \multicolumn{3}{|l|}{ Neurohypophysis } \\
\hline Normal site & $55(91.7 \%)$ & $8(24.3 \%)$ \\
\hline Ectopic site & $5(8.3 \%)$ & $25(75.7 \%)$ \\
\hline \multicolumn{3}{|l|}{ Adenohypophysis } \\
\hline Normal size & 15 (25\%) & $9(27.3 \%)$ \\
\hline Hypoplasic size & 45 (75\%) & $24(72.7 \%)$ \\
\hline
\end{tabular}

the associated hormone deficiencies were confirmed during follow-up. In 17 of the 25 children with MRI anomalies and in 7 of the 8 patients without MRI abnormalities, severe pituitary hypoplasia was observed (Table 2). One-third of MPHD patients showed FSH and LH deficiency some years after the beginning of $\mathrm{GH}$ therapy, in particular 7 of the 25 children with MRI abnormalities and 3 of 8 subjects having no neuroradiological features. In MPHD patients, no hormonal recovery was found.

\section{Discussion}

A high frequency of morphological abnormalities of the hypothalamo-pituitary region revealed by MRI has been reported in GHD patients (1-7). Injection of contrast material also contributed to the recognition of more subtle anomalies of the stalk, i.e. in cases in which the stalk was absent, the neurohypophysis was found to be ectopic. The PSA and ectopia of the neurohypophysis may be considered as diagnostic markers of permanent GHD since they have been more frequently reported in MPHD than in IGHD patients $(2-7)$. On the other hand, both MRI abnormalities are not invariably associated with MPHD as they have been observed also in normal children (8). Moreover, the adenohypophysis may be evaluated in terms of its size. Severe anterior pituitary hypoplasia is often observed in MPHD patients with or without other neuroradiological abnormalities, and, even if less frequently, in IGHD children (1, 5-10). Therefore, a severe isolated hypoplasia of the adenohypophysis could be an additional valuable marker of GHD. However, its diagnostic value is limited by the variability of the gland shape among healthy children $(1,8)$. Nevertheless, a small pituitary gland does not distinguish prognostically between IGHD and MPHD children, except for pubertal patients as previously reported (3). The absence of the adenohypophysis is highly suggestive of $\operatorname{MPHD}(7,10)$. Interestingly, the development of MPHD in children who have typical MRI anomalies and apparently IGHD confirms the important role of neuroradiological evaluation in the diagnostic approach of hypopituitarism.
These children are at risk of developing additional hormone deficiencies. The diagnosis of MPHD may be underestimated until the time of puberty, because the GnRH test fails to diagnose gonadotrophin deficiency in prepuberty. However, MPHD subjects may show normal MRI features, suggesting that neuroradiological abnormalities cannot be considered as markers of permanent $\mathrm{GH}$ deficiency. Since the neuroradiological abnormalities of the hypothalamo-pituitary region are detected by MRI in only $12-20 \%$ of sporadic cases of GHD, many genetic defects such as Pit-1, Prop-1 and HESX-1 mutations may not be diagnosed (11-16). Our long-term follow-up indicates that the children with both PSA and ectopia of the neurohypophysis, and apparent IGHD will develop additional pituitary hormone deficiencies over time.

Interestingly, in GHD patients we found no correlations between the size of the pituitary gland and the severity of the endocrine defect.

In conclusion, IGHD patients showing PSA and PPE inevitably develop MPHD, while IGHD subjects having no MRI abnormalities maintain IGHD.

\section{References}

1 Fujisawa I, Asato R, Nishimura K, Togashi K, Itoh K, Nakano Y et al. Anterior and posterior lobe of the pituitary gland: assessment by $1.5 \mathrm{~T}$ MR imaging. Journal of Computer Assisted Tomography 198711 214-220.

2 Argyropoulou M, Perignon F, Brauner R \& Brunelle F. Magnetic resonance imaging in the diagnosis of growth hormone deficiency. Journal of Pediatrics 1992120 886-891.

3 Bozzola M, Adamsbaum C, Biscaldi I, Zecca M, Cisternino M, Genovese $\mathrm{E}$ et al. Role of resonance imaging in the diagnosis and prognosis of growth hormone deficiency. Clinical Endocrinology $19964521-26$.

4 Triulzi F, Scotti G, di Natale B, Pellini C, Lukezic M, Scognamiglio $\mathrm{M}$ et al. Evidence of a congenital midline brain anomaly in pituitary dwarfs: a magnetic resonance imaging study in 101 patients. Pediatrics 199493 409-416.

5 Adamsbaum C, Houang M, Andrè Ch, Richard I, Ginisty D, Chaussain JL et al. Imagerie par résonance magnétique des hypopituitarismes non tumoraux de l'enfant. Revue d'Imagerie Médicale 19935 251-257.

6 Negel BHP, Palmbach M, Petersen D \& Ranke MB. Magnetic resonance images of 91 children with different causes of short stature: pituitary size reflects growth hormone secretion. European Journal of Pediatrics 1997156 758-763.

7 Cacciari E, Zucchini S, Ambrosetto P, Tani G, Carlà G, Cicognani A et al. Empty sella in children and adolescents with possible hypothalamic-pituitary disorders. Journal of Clinical Endocrinology and Metabolism 199478 767-771.

8 Benshoff ER \& Katz BH. Ectopia of the posterior pituitary gland as a normal variant: assessment with MR imaging. American Journal of Neuroradiology $199011709-712$.

9 Barbeau C, Jouret B, Gallegos D, Sevely A, Manelfe C, Oliver I et al. Syndrome d'interruption de la tige pituitaire. Archives de Pédiatrie $19985274-279$.

10 Chen S, Léger J, Garel C, Hassan M \& Czernichow P. Growth hormone deficiency with ectopic neurohypophysis: anatomical variations and relationship between the visibility of the pituitary stalk asserted by magnetic resonance imaging and anterior pituitary function. Journal of Clinical Endocrinology and Metabolism $1999842408-2413$.

11 Radovick S, Cohen LE \& Wondisford FE. The molecular basis of hypopituitarism. Hormone Research 199849 (suppl 1) 30-36. 
12 Choen LE, Wondisford FE, Salvatoni A, Maghnie M, BruckerDavis F, Weintraub BD et al. A 'Hot Spot' in the Pit-1 gene responsible for combined pituitary hormone deficiency: clinica and molecular correlates. Journal of Clinical Endocrinology and Metabolism 199580 679-684.

13 Irie Y, Tatsumi K, Kusuda S, Kawawaki H, Boyages SC, Nose O et al. Screening for Pit-1 abnormality by PCR direct sequencing method. Thyroid 19955 207-211.

14 Ward L, Chavez M, Huot C, Lecocq P, Collu R, Décarie JC et al. Severe congenital hypopituitarism with low prolactin levels and age-dependent anterior pituitary hypoplasia: a clue to a Pit-1 mutation. Journal of Pediatrics 1998132 1036-1038.
15 Pellegrini-Bouiller I, Bélicar P, Barlier A, Gunz G, Charvet JP, Jaquet $\mathrm{P}$ et al. A new mutation of the gene encoding the transcription factor Pit-1 is responsible for combined pituitary hormone deficiency. Journal of Clinical Endocrinology and Metabolism $1996812790-2796$.

16 Pfäffle R, Kim C, Otten B, Wit JM, Eiholzen U, Heimann G et al. Pit-1: clinical aspects. Hormone Research 199645 (suppl 1) 25-28.

Received 1 February 2000

Accepted 7 July 2000 